\title{
Supplemental Information: Structures of hard phases in thermoplastic polyurethanes
}

\author{
Maxwell W. Terban, Raphaël Dabbous, Anthony D. Debellis, \\ Elmar Pöselt, Simon J. L. Billinge*
}




\section{Data analysis}
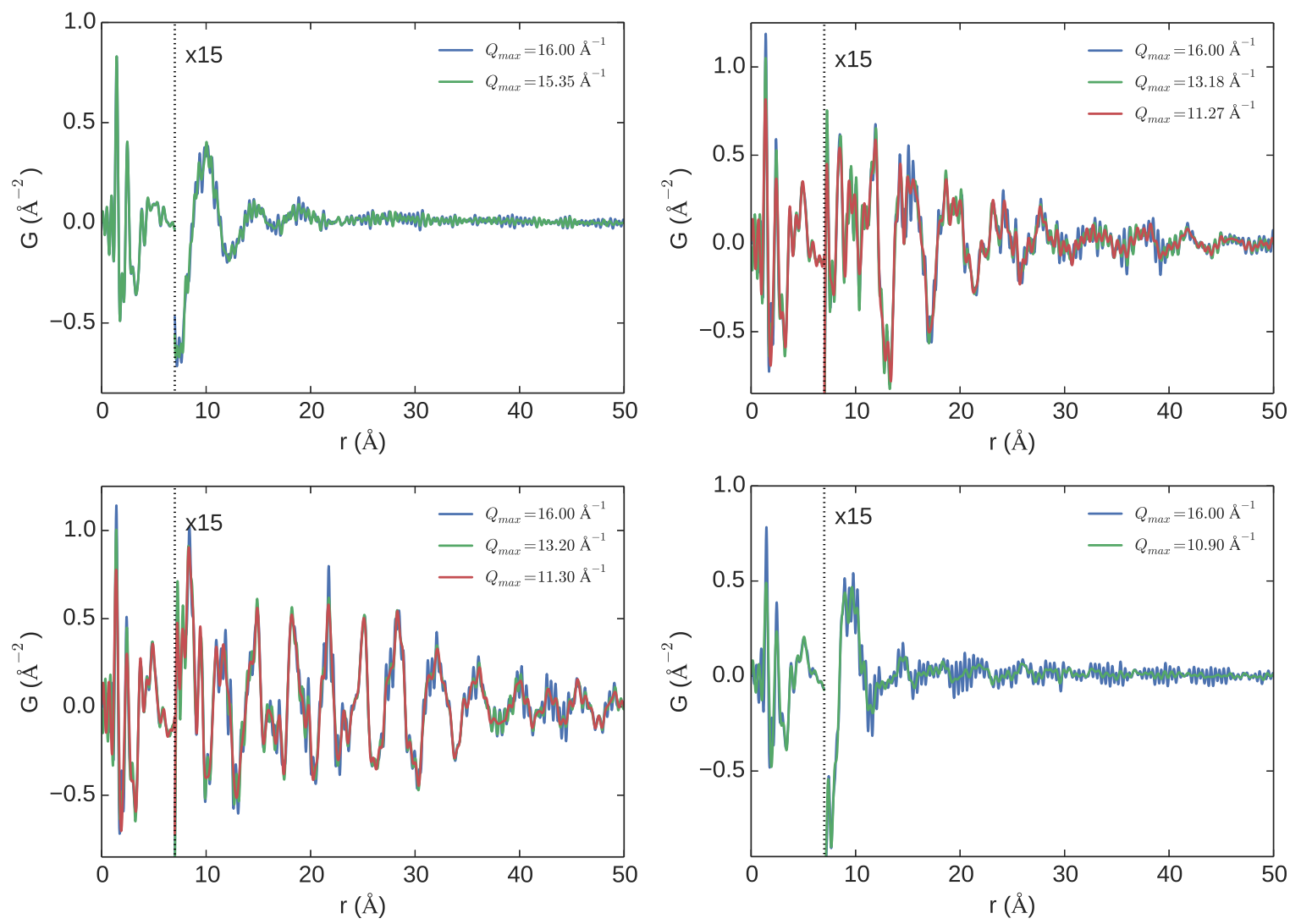

Figure 1: The $Q_{\max }$ used in the data reduction was varied depending on the region of scrutiny in order to optimize between real space resolution (high $Q_{\max }$ ) versus noise reduction (low $Q_{\max }$. We verified that the changes in this value did not degrade the signal of interest, particularly in the high-r region. Top left: HP1. Top right: HP3. Bottom left: HP4. Bottom right: TPU2. Low- and mid- $r$ refinements used $Q_{\max }=13.20 \AA^{-1}$ while high- $r$ refinements used $Q_{\max }=11.3 \AA^{-1}$ 

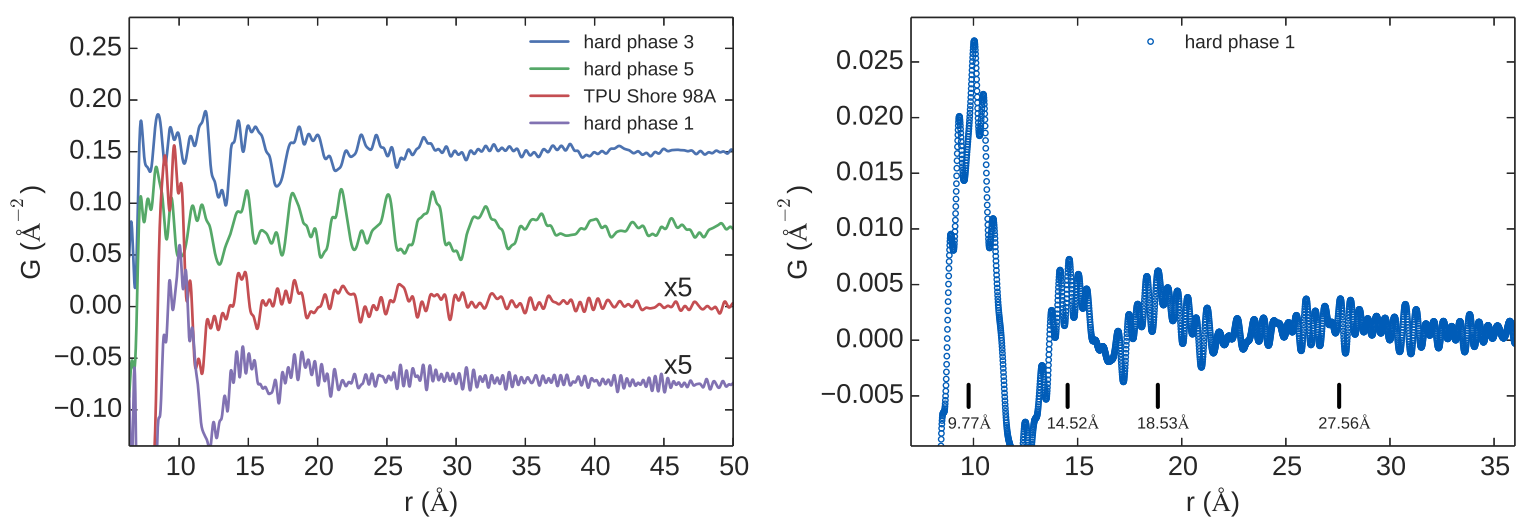

Figure 2: We highlight the high- $r$ signal in HP1 and TPU2. Left: The structuring in the PDFs of HP1 and TPU2 is compared to that of HP3 and HP5. It is clear that both samples show significant differences from the more crystalline phases. However, we can see that there is a small structural signal in TPU2 extending to higher- $r$ given the distinct peak shapes. Right: HP1 shows several features at 9.77, 14.52, 18.53, and 27.56 $\AA$. These features are much broader than the other sample PDFs indicating that there is far more structural disorder in samples HP1 and HP2.

A.

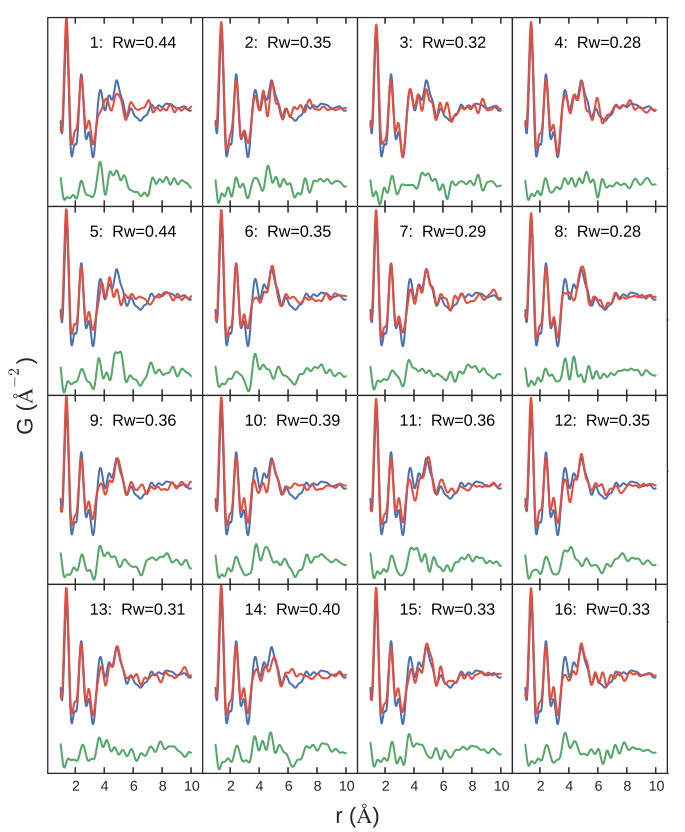

B.

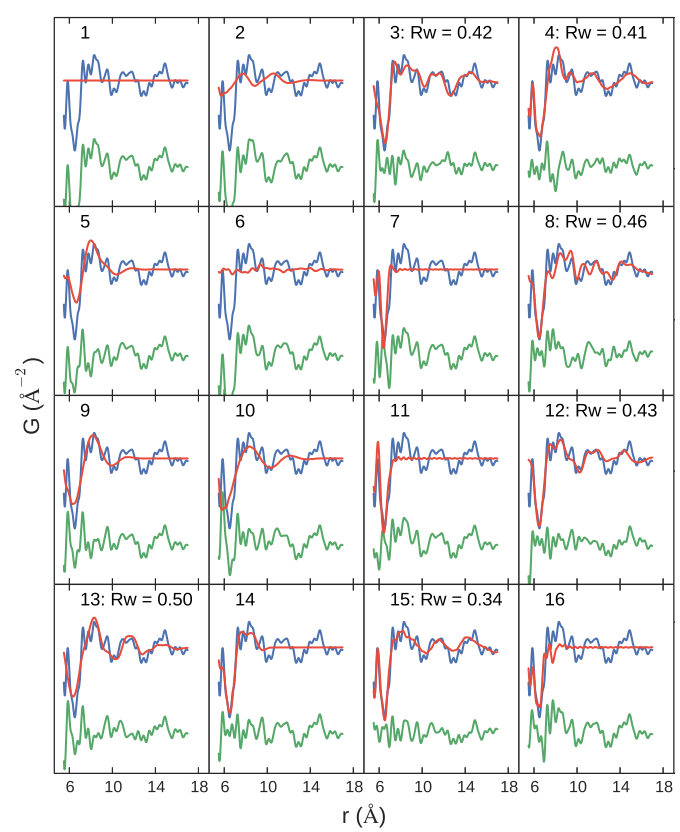

Figure 3: Measured PDF (blue symbols) of sample HP5 with fits of the 16 structural models (red lines) with the difference curve plotted below. (A) sixteen sub-panels show the fits to all models in the low- $r$ region and (B) fits to the intermediate- $r$ region. 

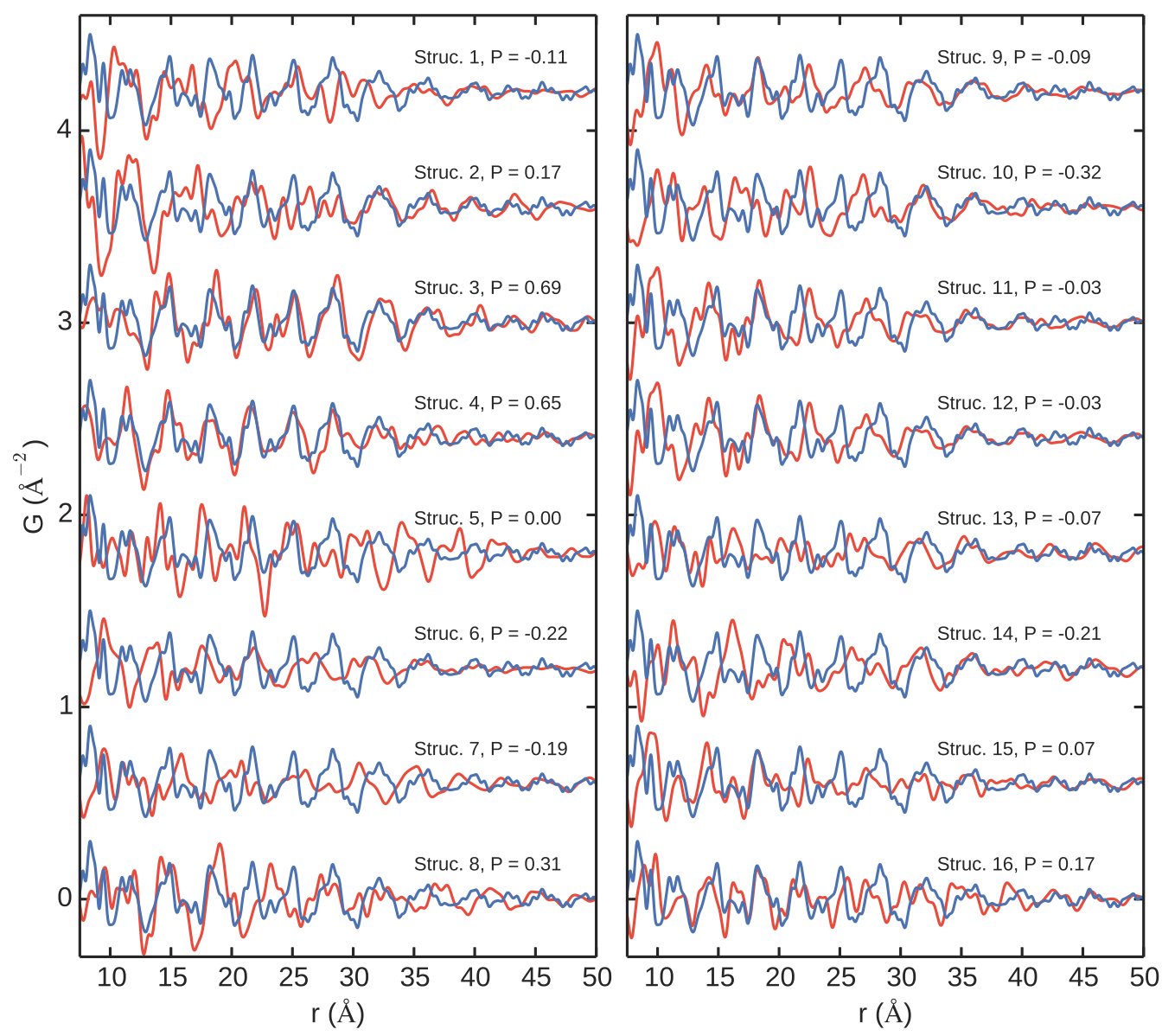

Figure 4: Comparison of measured PDF of HP5 (blue) $\left(Q_{\max }=11.3 \AA^{-1}\right)$, with models $1-16$ superimposed (red) for direct comparison. Pearson coefficients listed for reference. 

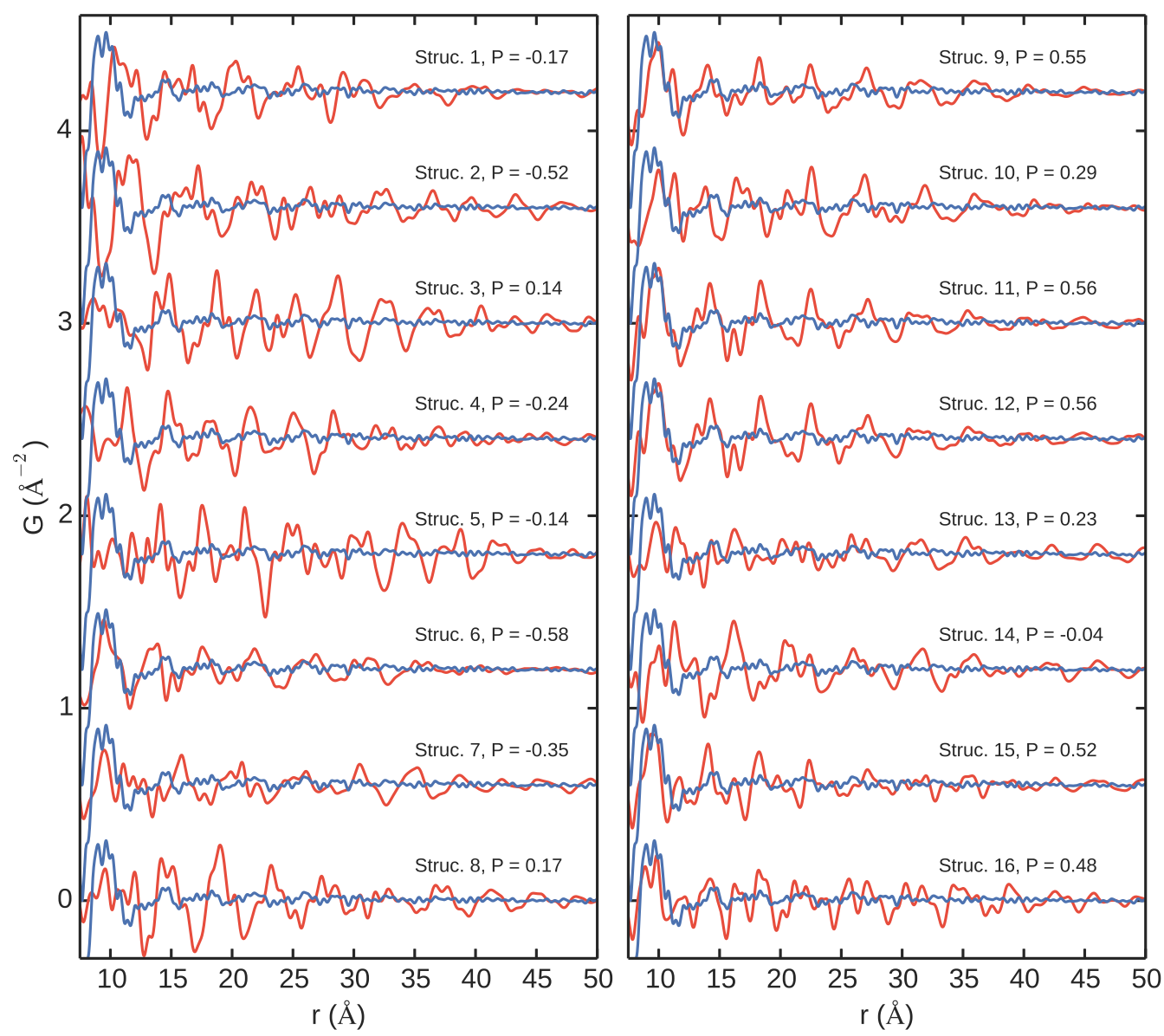

Figure 5: Comparison of measured PDF of TPU2 (blue) $\left(Q_{\max }=10.90 \AA^{-1}\right)$, with models $1-16$ superimposed (red) for direct comparison. Pearson coefficients listed for reference. We can see that the high- $r$ region does not correspond well with any of the models tested. Models 9, 11, 12,15 , and 16 show some similarities in the mid- $r$ region 


\section{Calibration standards and structure models}

Table 1: Measurements were performed on three separate dates at the X17A beamline. On each date, the detector geometry was calibrated by measuring a standard. The same standard was used to determine $Q_{d a m p}$ and $Q_{\text {broad }}$, the instrumental dampening and broadening of the PDF signal. [1]

\begin{tabular}{lcccc}
\hline Measurements & standard & detector distance $(\mathrm{mm})$ & $Q_{\text {damp }}\left(\AA^{-1}\right)$ & $Q_{\text {broad }}\left(\AA^{-1}\right)$ \\
\hline MDI and BDO & LaB6 & 204.2223 & 0.0401 & 0.0158 \\
TPU products & $\mathrm{Ni}$ & 206.1456 & 0.0390 & 0.0174 \\
hard phases 1-5 & $\mathrm{Ni}$ & 204.7059 & 0.0421 & 0.0188 \\
\hline
\end{tabular}


Table 2: 3D structure models used in the PDF analysis. Gauche and trans refer to the state of the central bond of the butane-diol fragment and the difference is shown in Fig. 6. 'Type' describes distinct differences in the chain packing perpendicular to the hydrogen bonding direction and is broken down into five types which are described below (examples shown in Fig. 7). Models 3, 4, and 5 are not polymerized models.

\begin{tabular}{llllllllll}
\hline$\#$ & model & S. G. & $a$ & $b$ & $c$ & $\alpha$ & $\beta$ & $\gamma$ & Type \\
\hline 1 & MDI/BDO (Born) [2] & $\mathrm{P} 2_{1} / \mathrm{b}$ & 5.103 & 39.628 & 6.735 & 85.948 & 90.0 & 90.0 & $\mathrm{~A} 0$ \\
2 & MDI/BDO (Sun) [3] & $\mathrm{P} 1$ & 5.33 & 5.26 & 38.68 & 113.6 & 116.0 & 94.4 & $\mathrm{~A} 0$ \\
3 & ${\text { BuOH-capped MDI [4] }{ }^{1}}$ & $\mathrm{P} 2_{1} 2_{2} 2$ & 16.511 & 13.970 & 4.778 & 90.0 & 90.0 & 90.0 .0 & \\
4 & MeOH-capped MDI 1 [5] $^{2}$ & $\mathrm{P} 112_{1} / \mathrm{b}$ & 5.157 & 9.800 & 31.472 & 90.0 & 90.0 & 93.90 & \\
5 & MeOH-capped MDI 2 [6] $^{3}$ & $\mathrm{Aba} 2$ & 8.167 & 16.54 & 11.56 & 90.0 & 90.0 & 90.0 & \\
6 & TPU-BDO-gauche $^{4}$ & $\mathrm{P} 1$ & 4.990 & 9.459 & 34.660 & 91.106 & 92.304 & 88.310 & $\mathrm{~B}$ \\
7 & TPU-BDO-trans & $\mathrm{P} 1$ & 4.860 & 10.070 & 33.617 & 89.109 & 91.777 & 90.377 & $\mathrm{~B}$ \\
8 & TPU-BDO-trans-3 & $\mathrm{P} 2_{1} / \mathrm{c}$ & 4.985 & 34.202 & 11.088 & 90.0 & 120.513 & 90.0 & $\mathrm{~A} 2$ \\
9 & TPU-BDO-gauche-1 & $\mathrm{P} 1$ & 5.066 & 9.952 & 34.246 & 91.178 & 92.062 & 89.398 & $\mathrm{~B}$ \\
10 & TPU-BDO-gauche-2 & $\mathrm{P} 1$ & 5.094 & 9.907 & 34.465 & 90.282 & 91.989 & 90.409 & $\mathrm{~A} 1$ \\
11 & TPU-BDO-gauche-3 & $\mathrm{P} 1$ & 5.062 & 10.042 & 33.833 & 91.753 & 91.319 & 89.639 & $\mathrm{C}$ \\
12 & TPU-BDO-gauche-4 & $\mathrm{P} 1$ & 5.060 & 10.056 & 33.827 & 91.706 & 91.488 & 89.910 & $\mathrm{C}$ \\
13 & TPU-BDO-trans-1 & $\mathrm{P} 1$ & 4.999 & 10.344 & 33.310 & 89.618 & 91.092 & 89.884 & $\mathrm{~B}$ \\
14 & TPU-BDO-trans-2 & $\mathrm{P} 1$ & 5.004 & 10.169 & 34.305 & 89.929 & 92.075 & 90.097 & $\mathrm{~A} 1$ \\
15 & TPU-BDO-trans-3-2 & $\mathrm{P} 2_{1} / \mathrm{c}$ & 5.051 & 34.493 & 11.167 & 90.0 & 117.140 & 90.0 & $\mathrm{~A} 2$ \\
16 & TPU-BDO-trans-4 & $\mathrm{P} 1$ & 4.968 & 10.602 & 32.294 & 89.947 & 90.099 & 90.015 & $\mathrm{C}$ \\
\hline
\end{tabular}

WebCSD codes ${ }^{1}$ BUPHCB, ${ }^{2}$ MEPHCB, ${ }^{3}$ MEPHCB01. ${ }^{4}$ Structures $6-16$ developed by A. D. Debellis

\section{Packing Types (direction perpendicular to hydrogen bonding):}

A0: Butane-diol and diphenyl-methane segments are in the same orientation from neighbor to neighbor. Chains stack vertically.

A1: Diphenyl-methane segments are in the same orientation from neighbor to neighbor, but butane-diol segments alternate. Chains stack vertically.

A2: Butane-diol segments are in the same orientation from neighbor to neighbor. Diphenylmethane segments stack vertically but with the orientations of the phenyl rings rotated from neighbor to neighbor.

B: Every other diphenyl-methane segment is oriented similar to the neighboring chain but the rest stack out of phase with different phenyl orientations.

C: Chains stack completely out of phase. All phenyl groups are rotated with repect to those on the neighboring chain. 

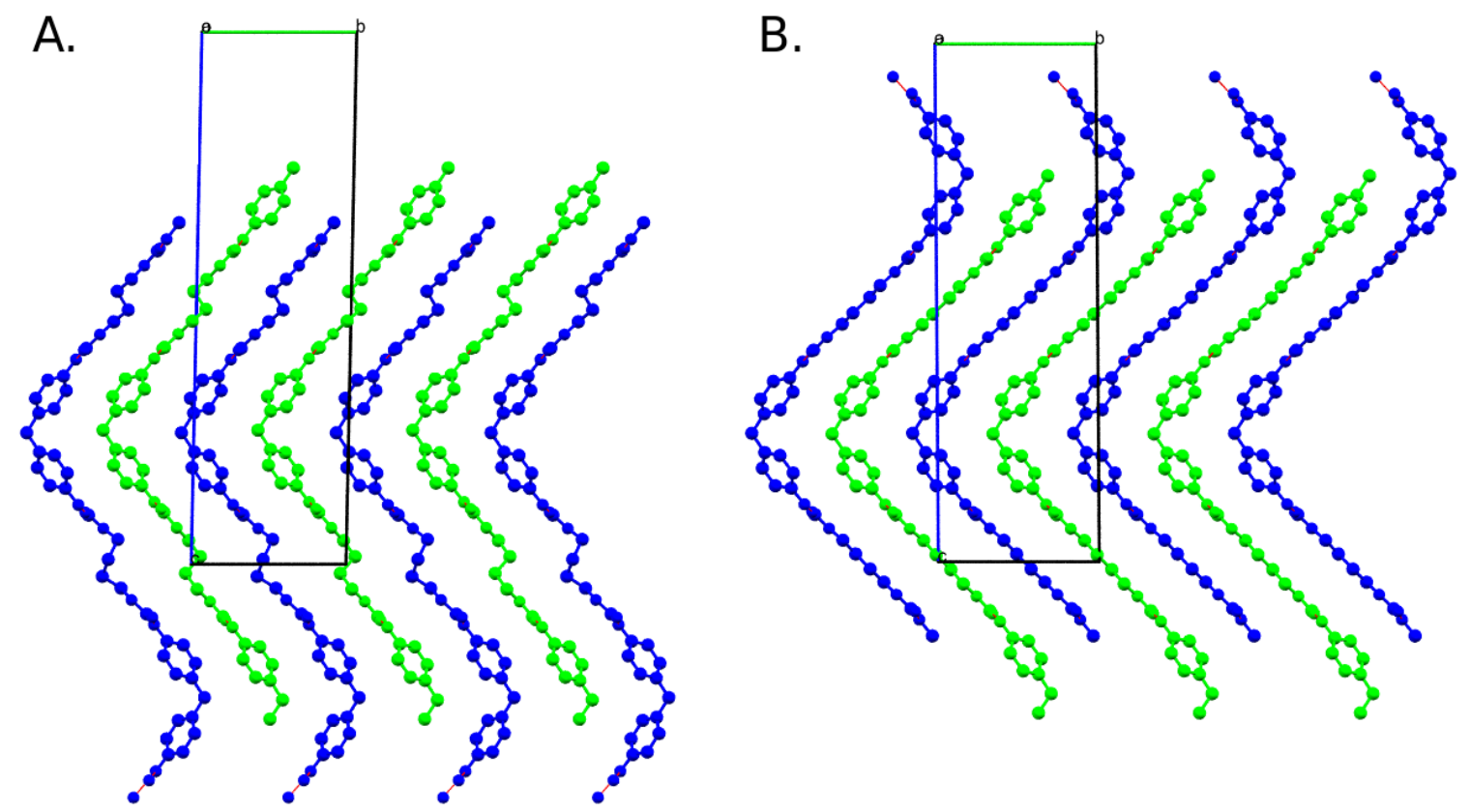

Figure 6: Comparison of (A) model 9 (TPU-BDO-gauche-1) and (B) model 13 (TPU-BDOtrans-1) in the [100] direction, parallel to the hydrogen bonding direction. The difference between gauche and trans bonding in the butane-diol fragment is evident. The fragment forms a kink in the former, while it forms in a zig-zag fashion in the latter which remains fairly linear. Looking in the direction parallel to hydrogen bonding, all the models look similar to these examples depending on whether they are gauche or trans. 

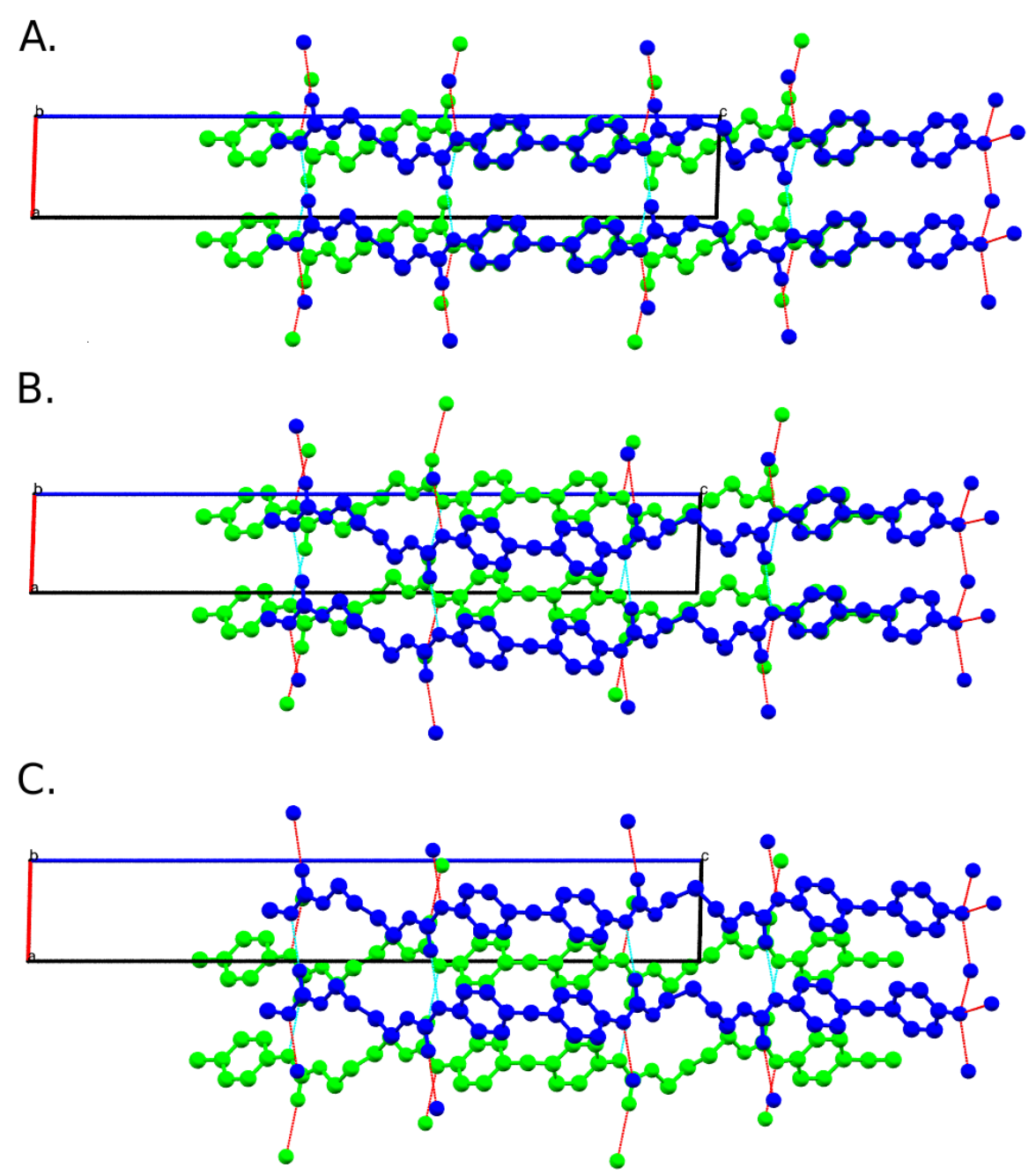

Figure 7: Comparison of models (A) 10 (TPU-BDO-gauche-2, type A1), (B) 9 (TPU-BDOgauche-1, type B), and (C) 11 (TPU-BDO-gauche-3, type C) in the [010] direction, perpendicular to the hydrogen bonding direction. Of the new models developed and tested, these represent the essentially three different varieties of chain packing in this direction. In (A), the diphenyl-methane segments stack in the same orientation, while the butane-diol segment switches between two orientations from neighbor to neighbor. In (B), only every other diphenylmethane segment stacks in the same orientation, while the others shift out of phase in the [100] direction. In (C), all segments are out of phase from layer to layer in this direction.

\section{Correlation Analysis}

Models were calculated with a thermal parameter $U_{\text {iso }}=0.035 \AA^{2}$, a sharpening coefficient $\delta_{2}=1.0 \AA^{2}$, and a $Q_{\max }=11.3 \AA^{-1}$. The $Q_{\max }$ was chosen in order to reduce the ripples in $G(r)$ coming from noise at high- $Q$ in the measured data. The Pearson product-momentum 
correlation coefficient is calculated by

$$
r=\frac{1}{1-n} \sum_{i=0}^{n}\left(\frac{X_{i}-\bar{X}}{\sigma_{x}}\right)\left(\frac{Y_{i}-\bar{Y}}{\sigma_{y}}\right),
$$

where $\bar{X}$ and $\bar{Y}$ are the means and $\sigma_{x}$ and $\sigma_{y}$ are the standard deviations of the respective datasets. This calculation gives a value between -1 and 1 where -1 implies anticorrelation, 0 implies no correlation, and 1 implies perfect correlation. This is a good value to benchmark the likeness and therefore structural similarity between the PDF curves $[7,8]$, see Table 3.

Table 3: Pearson product-momentum correlation coefficient values between $G(r)$ signals of the TPU hard phases (HP) and products calculated over a range of 7.5-50.0 . Values of 0.8 or higher are in bold to highlight datasets with a high degree of similarity.

\begin{tabular}{lccccccc}
\hline Samples & h.p. 1 & h.p. 2 & h.p. 3 & h.p. 4 & h.p. 5 & TPU60D & TPU98A \\
\hline HP1 & 1.0000 & $\mathbf{0 . 9 6 1 7}$ & 0.2129 & 0.026 & -0.1681 & $\mathbf{0 . 8 2 8 5}$ & $\mathbf{0 . 8 4 3 8}$ \\
HP2 & - & 1.0000 & 0.2148 & 0.0127 & -0.1811 & 0.7511 & 0.7669 \\
HP3 & - & - & 1.0000 & $\mathbf{0 . 8 6 6 1}$ & 0.3841 & 0.1002 & 0.1042 \\
HP4 & - & - & - & 1.0000 & 0.553 & 0.0461 & 0.0369 \\
HP5 & - & - & - & - & 1.0000 & -0.1196 & -0.1171 \\
TPU60D & - & - & - & - & - & 1.0000 & $\mathbf{0 . 9 8 5 0}$ \\
tpu98A & - & - & - & - & - & - & 1.0000 \\
\hline
\end{tabular}

\section{Crystallite Domain Size}

For nano-sized crystallites, the attenuation of the PDF signal is directly related to the size and shape of the domain of structural coherence, approximately the crystallite size, though likely smaller for molecular materials due to various types of disorder and thermal motion. This size effect is quantitatively accounted for by modulating an infinite crystalline PDF signal with a characteristic function $\gamma(r)[9]$.

$$
G_{\text {nano }}(r)=\gamma(r) G_{\infty}(r)
$$

which reflects the expected attenuation with increasing- $r$. The characteristic function for a sphere is

$$
\gamma(r)_{\text {sphere }}=\left[1-\frac{3 r}{2 d}+\frac{1}{2}\left(\frac{r}{d}\right)^{3}\right] H(d-r),
$$

where $d$ is the domain diameter. $H(r)$ is a step function with value 1 for $r \leq d$ and 0 beyond. [10] The signal is additionally attenuated by the finite resolution of the measurement [11]; this is accounted for by obtaining an instrumental damping value $Q_{\text {damp }}$ from a standard measurement as noted in Table 1. 


\section{$5 \quad$ Model refinements}

Table 4: Refined values for structural parameters from hard phase refinements.

\begin{tabular}{lcccc}
\hline & Model I & Form I & Model II & Form II \\
\hline Space group & $\mathrm{P} 2_{1} / \mathrm{c}$ & & $\mathrm{P} 2_{1} 2_{1} 2$ & \\
$a(\AA)$ & 4.9845 & 5.022 & 16.511 & 16.253 \\
$b(\AA)$ & 34.2020 & 32.707 & 13.970 & 13.871 \\
$c(\AA)$ & 11.0880 & 11.508 & 4.778 & 4.749 \\
$\beta(\mathrm{deg})$ & 120.5128 & 119.137 & - & - \\
$\mathrm{U}_{\text {intra }}\left(\AA^{2}\right)$ & - & 0.005 & - & 0.010 \\
$\mathrm{U}_{\text {inter }}\left(\AA^{2}\right)$ & - & 0.043 & - & 0.053 \\
crystalline phase $(\%)$ & - & 22 & - & 15 \\
crystalline phase $d_{c}(\AA)$ & - & 85 & - & - \\
disordered phase $d_{c}(\AA)$ & - & 18 & - & 20 \\
$R_{w}$ & - & 0.23 & - & 0.33 \\
\hline
\end{tabular}



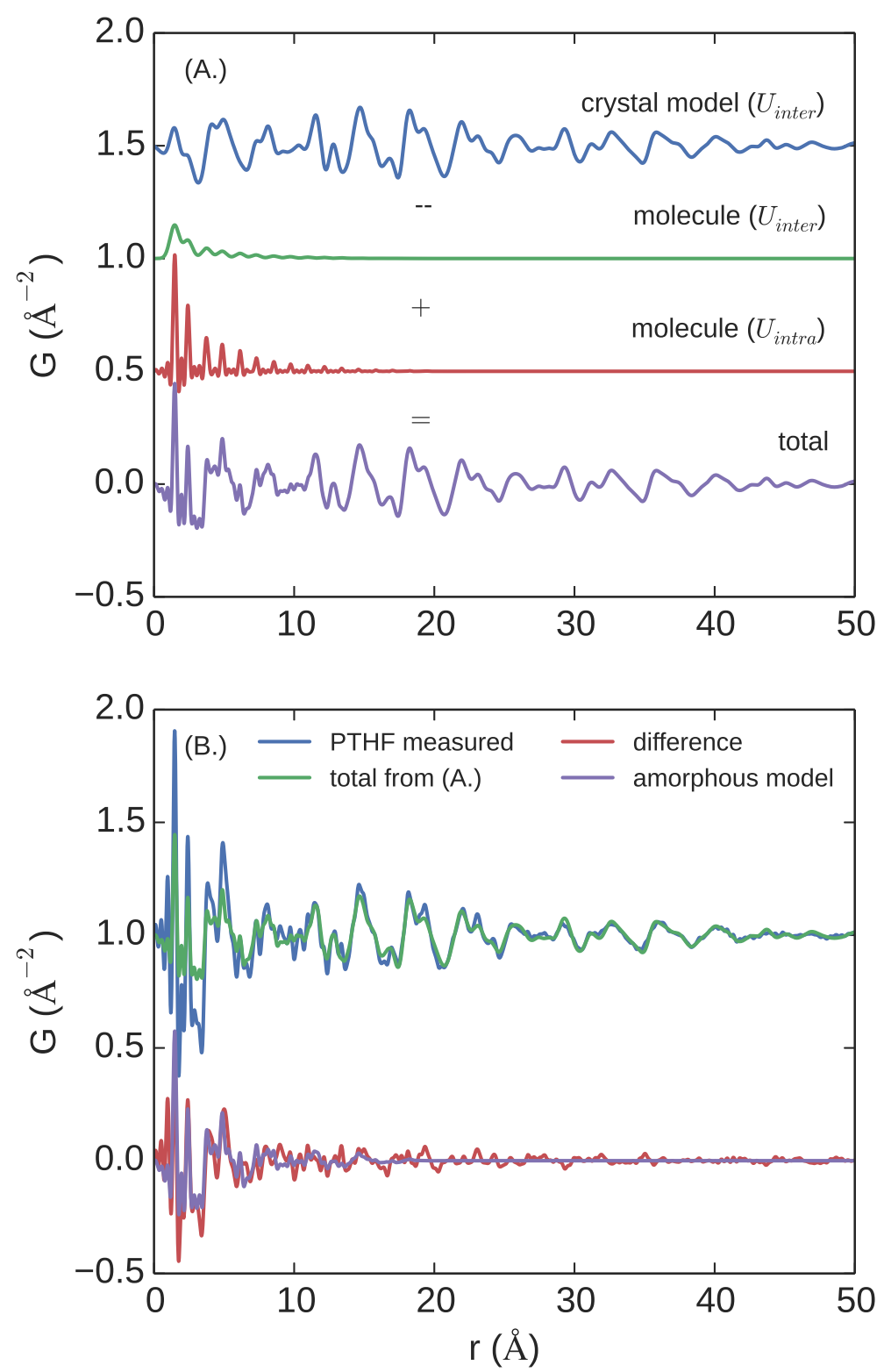

Figure 8: Depiction of process for fitting the model structures to the data, shown for the case of PTHF. (A) The crystal model is simulated and fit to the high- $r$ region of the measured data to determine the intermolecular thermal parameter, $U_{\text {inter }}$. The PDF of a single molecule from the same structure is then calculated with the same thermal parameter and subtracted to obtain the intermolecular PDF. Another PDF of the molecule is then calculated with a much lower thermal parameter $U_{\text {intra }}$ and added to produce the sharp peaks seen in the low- $r$ region. (B) The full PDF produced from (A) is not able to describe the significant attenuation seen going from the low- $r$ to high- $r$ regions, indicating an additional disordered phase is present. This phase is modeled with the same local structure as the crystalline phase but calculated with a much smaller crystallite size. 


\section{References}

[1] Proffen, T.; Billinge, S. J. L. PDFFIT, a program for full profile structural refinement of the atomic pair distribution function. J. Appl. Crystallogr. 1999, 32, 572-575

[2] Born, L.; Crone, J.; Hespe, H.; Müller, E. H.; Wolf, K. H. On the structure of polyurethane hard segments based on MDI and butanediol-1,4: X-ray diffraction analysis of oriented elastomers and of single crystals of a model compound. J. Polym. Sci. Pol. Phys. 1984, 22, 163-173

[3] Sun, Z. X-ray analysis of structures of polyurethanes and copolyesters. Case Western Reserve University, Cleveland, OH, 1990, electronic Thesis.

[4] Forcier, P. G.; Blackwell, J. The structure of bis(4-hydroxybutyl) 4,4'methylenebis(phenylcarbamate): a model compound for diol-linked MDI units in polyurethane elastomers. Acta Crystallogr. B 1981, 37, 1286.

[5] Gardner, K. H.; Blackwell, J. Structure of dimethyl 4,4'-methylenebis(phenylcarbamate): a model for MDI units in polyurethane hard segments. Acta Crystallogr. B 1980, 36, $1972-1975$

[6] Born, L.; Hocker, J.; Paulus, H.; Wolfel, E. The stable modification (modification-2) of (Methylene-di-4,1-phenylene)biscarbamic acid dimethylester C17H18N2O4. Acta Crystallogr. C 1981, 10, 187-190

[7] Myers, J. L.; Well, A. D. Research Design and Statistical Analysis. Hillsdale: Lawrence Erlbaum Associates, 3 edition, 2010.

[8] Dykhne, T.; Taylor, R.; Florence, A.; Billinge, S. J. L. Data requirements for the reliable use of atomic pair distribution functions in amorphous pharmaceutical fingerprinting. Pharmaceut. Res. 2011, 28, 1041-1048

[9] Kodama, K.; Iikubo, S.; Taguchi, T.; Shamoto, S. Finite size effects of nanoparticles on the atomic pair distribution functions. Acta Crystallogr. A 2006, 62, 444-453

[10] Guinier, A.; Fournet, G.; Walker, C.; Yudowitch, K. Small-angle scattering of x-rays. John Wiley \& Sons, Inc., New York, 1955.

[11] Egami, T.; Billinge, S. J. L. Underneath the Bragg peaks: structural analysis of complex materials. Elsevier, Amsterdam, 2nd edition, 2012. 